\title{
The Safety and Efficacy of Primary Duct Closure without Endoscopic Nasobiliary Drainage after Laparoscopic Common Bile Duct Exploration
}

\author{
Yan Yang Lin Han Da-Ning Lin Zeng-Ji Hu Wei Tu Feng Chen \\ Yong-Qiang Li
}

Department of Hepatobiliary Pancreatic Surgery, The First Affiliated Hospital of Hainan

Medical University, Haikou, China

\section{Keywords}

Choledocholithiasis · Choledochoscopy · Endoscopic retrograde cholangiopancreatography · Suture $\cdot$ Complications

\begin{abstract}
Background: Primary duct closure (PDC) after laparoscopic common bile duct exploration (LCBDE) has been widely applied for choledocholithiasis. However, there has been controversy over the placement of endoscopic nasobiliary drainage (ENBD) during operation. To date, few studies compare the clinical effect of PDC without and with ENBD. The aim of this study was to assess the safety and efficacy of PDC without ENBD for choledocholithiasis. Methods: From January 2016 to December 2018, a total of 164 patients meeting the inclusion criteria were enrolled and divided into group A (undergone LCBDE + PDC without ENBD, 81 cases) and group B (undergone LCBDE + PDC with ENBD, 83 cases) in this study. The intraoperative conditions and postoperative complications were compared between the 2 groups. Results: In group A, the time of operation, postoperative first flatus, extubation, antibiotics, and discharge were shorter than in group $\mathrm{B}(t=-17.775, p=0.000 ; t=-7.649, p=0.000 ; t=$ $-5.807, p=0.000 ; t=-9.247, p=0.000 ; t=-9.322, p=0.000$, respectively). Furthermore, intraoperative blood loss was less $(t=-2.199, p=0.029)$ and hospital costs were lower $(t=$ $-6.685, p=0.000$ ). However, there was no significant difference in postoperative complications between the 2 groups ( $p>0.05$ ). Conclusions: In patients who meet the screening criteria, PDC without ENBD after LCBDE is safe and effective and worthy of clinical application.
\end{abstract}




\section{Introduction}

Choledocholithiasis is a common disease in biliary surgery and relates to severe complications such as cholangitis or pancreatitis [1]. In recent years, with innovation and development in minimally invasive surgical techniques, laparoscopic common bile duct exploration (LCBDE) plus primary duct closure (PDC) have been widely adopted because of the efficacy and safety [2, 3]. Estellés Vidagany et al. [4] reported that the success rate for stone clearance was $96.2 \%$, in addition to the mortality rate and CBD stenosis rate being $0 \%$. However, the overall complication rate was $15 \%$ and the biliary complication rate was $7.5 \%$; for example, bile leakage was $6.8 \%$. So, intraoperative endoscopic nasobiliary drainage (ENBD) through endoscopic retrograde cholangiopancreatography (ERCP) has been proposed. But, it is well known that serious complications of this operation include acute pancreatitis, sepsis, bleeding, cholangitis, duodenal perforation, and so on $[5,6]$. To date, whether ENBD following LCBDE is necessary for every patient remains a controversial issue [7]. Hence, the aim of this study was to assess the clinical efficacy of PDC with and without ENBD.

\section{Materials and Methods}

\section{Patients}

The patients with choledocholithiasis were undergone LCBDE at the Department of Hepatobiliary Pancreatic Surgery, the First Affiliated Hospital of Hainan Medical University, Haikou, Hainan, China. The inclusion criteria were based on the following $[8,9]$ : (1) stones can be cleared within one procedure, $(2)<5$ stones in CBD, (3) stone size $\leq 1.5 \mathrm{~cm}$, (4) CBD diameter $\geq 0.8 \mathrm{~cm}$, and (5) good functional sphincter of Oddi. The exclusion criteria included the following [10,11]: (1) intrahepatic bile duct stones, (2) bile duct stricture, (3) acute obstructive suppurative cholangitis, (4) duodenal papilla stenosis or occlusion, (5) accompanying with malignant tumor, (6) biliary pancreatitis, and (7) severe systemic disease leading to inability to tolerate surgery. All patients were identified and assigned to 2 groups: LCBDE plus PDC without ENBD (group A, $n=$ 81) and with ENBD (group B, $n=83$ ). The ethical approval was obtained from the First Affiliated Hospital of Hainan Medical University Ethics Committee (Grant No. 2016 [scientific research] No [3]), and written informed consents were obtained from all patients.

\section{Surgery}

All surgical approaches were performed by the same surgical team with 4-port [12]. In both groups, after removing the gallbladder, a longitudinal incision of $10 \sim 15 \mathrm{~mm}$ was made in the anterior aspect of the CBD, and a flexible choledochoscope (Olympus-P60; Olympus, Tokyo, Japan) was used to detect the stones. A stone basket (Wilson-Cook MWB3*6; Winston-Salem, NC, USA) was applied to extract CBD stones through the working channel of the choledochoscope. When all stones were cleared, the basket could be successfully passed through the duodenal papilla.

Next, in group B, a zebra guide wire (MTW-0.027; MTW Endoskopie, Wesel, Germany) was inserted into the CBD via an intraoperative choledochoscope and retrieved by the intraoperative ERCP (Olympus CV-260; Olympus, Tokyo, Japan). And then, under the guidance of the zebra guide wire, a nasobiliary drainage tube (Wilson-Cook 8.5F; Winston-Salem, NC, USA) was placed into the CBD, in which the head of the tube was at least $1 \mathrm{~cm}$ above the CBD incision and the end was dragged out from the nose. Finally, in both groups, the CBD was interruptedly or continuously closed with 4-0 Ethicon absorbable suture (Johnson \& Johnson, West Somerville, NJ, USA), and a $20 \mathrm{~F}$ silica gel drainage tube was routinely placed beside the foramen of Winslow (Fig. 1).

\section{Outcome Definition}

Postoperative morbidity was the number of complications occurring within the first 30 days after surgery, and complications were grouped according to the Dindo-Clavien classification [13]. Postoperative mortality was the number of deaths occurring during the first 30 days after surgery. Bile leakage was defined and classified according to the International Study Group of Liver Surgery [14]. A retained stone was a stone detected in the CBD during the first 2 years after surgery [15]. 

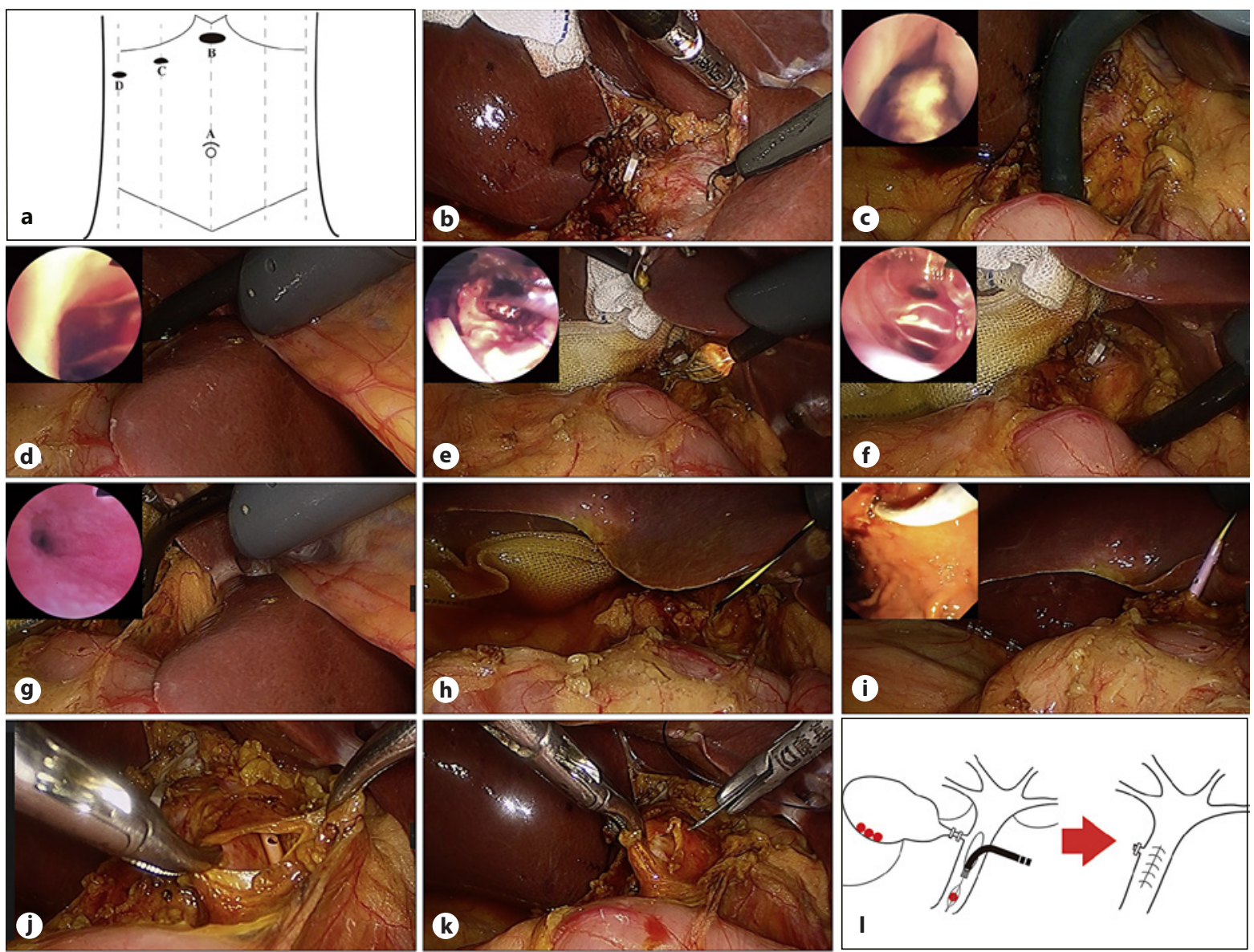

Fig. 1. Intraoperative photographs showing the surgical process of LCBDE plus PDC with or without ENBD. a Showing the position of trocars. b Choledochotomy. c Exploring the CBD by the choledochoscope. d, e Extracting the stone. $\mathbf{f}, \mathbf{g}$ Exploring the CBD once more. $\mathbf{h}$ Inserting the guide wire. $\mathbf{i}, \mathbf{j}$ Placing ENBD. $\mathbf{k}$ Closing the CBD at the first stage. I Showing LCBDE and PDC. LCBDE, laparoscopic common bile duct exploration; PDC, primary duct closure; ENBD, endoscopic nasobiliary drainage.

\section{Statistical Analysis}

Data were statistically analyzed by using the software package SPSS (version 19.0) for Windows. Measurement data with normal distribution were represented as $\bar{x} \pm S D$. Comparison between the groups was analyzed by Student's $t$ test. Comparisons of count data were analyzed using the $\chi^{2}$ test or Fisher exact probability. A level of $p<0.05$ was considered statistically significant.

\section{Results}

\section{Demographics of Cohort}

From January 2016 to December 2018, a total of 164 eligible patients were undergone the surgery, and the patient characteristics are described in Table 1. Eight-six female and 78 male patients, with a median age of 60 years (range $21 \sim 88$ years), were included in this study. There were no significant differences in terms of demographic or clinical characteristics between the 2 groups $(p>0.05)$. The mean number and size of stones in the 2 groups were $1.96 \pm 1.07$ versus $1.65 \pm 1.13(t=1.819, p=0.071)$ and $0.87 \pm 0.36$ versus $0.81 \pm 0.33 \mathrm{~cm}$ $(t=1.125, p=0.262)$, respectively. The mean diameter of CBD was $1.26 \pm 0.34$ versus $1.17 \pm$ 
Table 1. Perioperative general characteristics of the 2 groups

\begin{tabular}{|c|c|c|c|c|}
\hline Characteristics & $\begin{array}{l}\text { Group A } \\
(n=81)\end{array}$ & $\begin{array}{l}\text { Group B } \\
(n=83)\end{array}$ & $\chi^{2} / t$ & $p$ values \\
\hline Age, years & $57.28 \pm 14.94$ & $57.55 \pm 16.35$ & -0.110 & 0.912 \\
\hline Sex (male/female) & $37 / 44$ & $41 / 42$ & $0.227^{\mathrm{a}}$ & 0.634 \\
\hline \multicolumn{5}{|l|}{ Presentations } \\
\hline Fever & $15(18.52 \%)$ & 25 (30.12\%) & $2.992^{\mathrm{a}}$ & 0.084 \\
\hline RUQ pain & $73(90.12 \%)$ & $78(93.98 \%)$ & $0.834^{\mathrm{a}}$ & 0.361 \\
\hline Jaundice & $39(48.15 \%)$ & $42(50.60 \%)$ & $0.099^{\mathrm{a}}$ & 0.753 \\
\hline \multicolumn{5}{|l|}{ Stone characteristics } \\
\hline Stones, $n$ & $1.96 \pm 1.07$ & $1.65 \pm 1.13$ & 1.819 & 0.071 \\
\hline Size of stones, $\mathrm{cm}$ & $0.87 \pm 0.36$ & $0.81 \pm 0.33$ & 1.125 & 0.262 \\
\hline Diameter of CBD, cm & $1.26 \pm 0.34$ & $1.17 \pm 0.35$ & 1.703 & 0.090 \\
\hline \multicolumn{5}{|l|}{ ASA score } \\
\hline 1 & 74 & 72 & $0.892^{\mathrm{a}}$ & 0.345 \\
\hline 2 & 7 & 11 & & \\
\hline 3 & 0 & 0 & & \\
\hline 4 & 0 & 0 & & \\
\hline 5 & 0 & 0 & & \\
\hline 6 & 0 & 0 & & \\
\hline Conversion to open surgery, \% & 0 & 0 & & \\
\hline Follow-up, months & $16.52 \pm 9.30$ & $17.64 \pm 10.30$ & -0.730 & 0.466 \\
\hline
\end{tabular}

RUQ, right upper quadrant; WBC, white blood cell; CBD, common bile duct; ASA, American Society of Anesthesiologists. ${ }^{a} \chi^{2}$ value.

Table 2. Outcomes of the 2 groups

\begin{tabular}{lcccc}
\hline Outcomes & $\begin{array}{l}\text { Group A } \\
(n=81)\end{array}$ & $\begin{array}{l}\text { Group B } \\
(n=83)\end{array}$ & $\chi^{2} / t$ & $p$ values \\
& & & & \\
\hline Closure methods & & & & \\
$\quad$ Interrupted suture & $32(39.51 \%)$ & $30(36.14 \%)$ & $0.197^{\mathrm{a}}$ & 0.657 \\
$\quad$ Continuous suture & $49(60.49 \%)$ & $53(63.86 \%)$ & & \\
Suture materials & & & & \\
$\quad$ Absorbable & $73(90.12 \%)$ & $83(100 \%)$ & $8.618^{\mathrm{a}}$ & 0.003 \\
$\quad$ Nonabsorbable & $8(9.88 \%)$ & $0(0 \%)$ & & \\
Operation time, min & $123.46 \pm 27.53$ & $192.00 \pm 21.57$ & -17.775 & 0.000 \\
Intraoperative bleeding, mL & $39.94 \pm 39.39$ & $50.14 \pm 15.22$ & -2.199 & 0.029 \\
Postoperative first flatus, day & $2.06 \pm 1.46$ & $4.10 \pm 1.91$ & -7.649 & 0.000 \\
Intraperitoneal drainage extubation, day & $4.46 \pm 2.73$ & $7.02 \pm 2.93$ & -5.807 & 0.000 \\
Postoperative antibiotic therapy, day & $5.95 \pm 2.64$ & $10.39 \pm 3.44$ & -9.247 & 0.000 \\
Postoperative hospital stay, day & $6.99 \pm 2.55$ & $11.55 \pm 3.62$ & -9.322 & 0.000 \\
Cost (¥, ten thousand yuan) & $4.84 \pm 0.83$ & $5.72 \pm 0.86$ & -6.685 & 0.000 \\
\hline \multicolumn{1}{c}{ a $\chi^{2}$ value. } & & & & \\
\hline
\end{tabular}

$0.35 \mathrm{~cm}(t=1.703, p=0.090)$. The median follow-up was 17 months (range 7-28 months) after surgery.

\section{Surgical Procedure}

All patients successfully received laparoscopic surgical therapy without converting to open surgery, and no postoperative mortality was found in any groups. During surgery, PDC 
Table 3. Perioperative complications and management of the 2 groups

\begin{tabular}{llllll}
\hline $\begin{array}{l}\text { Dindo-Clavien } \\
\text { classification }^{1}\end{array}$ & Complication & $\begin{array}{l}\text { Group A } \\
(n=81)\end{array}$ & $\begin{array}{l}\text { Group B } \\
(n=83)\end{array}$ & $p$ value $^{3}$ & Management \\
\hline I & Bile leakage & & & \\
& Grade A & 1 & 1 & 1.000 & Observation \\
& Grade B & 0 & 1 & 1.000 & Observation \\
& Wound infection & 1 & 1 & 1.000 & Dressing change \\
\hline II & Pneumonia & 1 & 4 & 0.367 & Antibiotics \\
& Acute pancreatitis & 1 & 0 & 0.494 & Octreotide \\
& Cholangitis & 4 & 4 & 1.000 & Antibiotics \\
& Hemobilia & 1 & 3 & 0.620 & Octreotide + hemostatic \\
\hline III $_{\mathrm{a}}$ & Bile leakage & & & & ERCP \\
\hline Grade B & 3 & 0 & 0.118 & \\
\hline IV & & 0 & 0 & & \\
\hline
\end{tabular}

ERCP, endoscopic retrograde cholangiopancreatography. ${ }^{1}$ Postoperative complications were grouped according to the Dindo-Clavien classification [13]. ${ }^{2}$ The definition and classification of bile leakage as provided by the International Study Group of Liver Surgery [14]. ${ }^{3}$ Fisher's exact test.

was performed in $39.51 \%$ (32/81) by interrupted sutureand in $60.49 \%$ (49/81) by continuous suture in group A, while $36.14 \%(30 / 83)$ and $63.86 \%(53 / 83)$ in group B $\left(\chi^{2}=0.197, p=\right.$ $0.657)$, in addition to $90.12 \%(73 / 81)$ by absorbable suture in group $A$ and $100 \%(83 / 83)$ in group $B\left(\chi^{2}=8.618, p=0.003\right)$. In both groups, the mean operation time was $123.46 \pm 27.53$ versus $192.00 \pm 21.57 \mathrm{~min}(t=-17.775, p=0.000)$ and the median intraoperative bleeding was $39.94 \pm 39.39$ versus $50.14 \pm 15.22 \mathrm{~mL}(t=-2.199, p=0.029)$ (Table 2).

\section{Postoperative Outcomes}

After surgery, the time of first flatus, intraperitoneal drainage extubation, antibiotic therapy, hospital stay, and the costs were $2.06 \pm 1.46$ days, $4.46 \pm 2.73$ days, $5.95 \pm 2.64$ days, $6.99 \pm 2.55$ days, and $4.84 \pm 0.83$ ten thousand yuan in group A, compared with $4.10 \pm 1.91$ days, $7.02 \pm 2.93$ days, $10.39 \pm 3.44$ days, $11.55 \pm 3.62$ days, and $5.72 \pm 0.86$ ten thousand yuan in group B, respectively. Statistically significant differences between the 2 groups were present in each of these parameters $(t=-7.649, p=0.000 ; t=-5.807, p=0.000 ; t=-9.247$, $p=0.000 ; t=-9.322, p=0.000 ; t=-6.685, p=0.000$ ) (Table 2).

\section{Postoperative Complications}

The overall postoperative morbidity rate was 14.81\% (12/81) in group A and 16.87\% $(14 / 83)$ in group B. The differences were not statistically significant $\left(\chi^{2}=0.129, p=0.719\right)$. Cases with grade A bile leakage, grade B bile leakage, cholangitis, hematobilia, acute pancreatitis, pneumonia, and wound infection were 1 (1.23\%), 3 (3.70\%), 4 (4.94\%), 1 (1.23\%), 1 (1.23\%), 1 (1.23\%), and $1(1.23 \%)$ in group A, compared with $1(1.20 \%), 1(1.20 \%), 4(4.82 \%)$, $3(3.61 \%), 0(0 \%), 4(4.82 \%)$, and $1(1.20 \%)$ in group B, and there was no significant difference between the 2 groups ( $p>0.05$ ) (Table 3). According to the Dindo-Clavien classification [13], most of the complications were grades I and II. Moreover, 3 patients with grade A or B bile leakage stopped spontaneously in 5 13 days through effective peritoneal drainage. However, in group A, there were 3 other patients with grade B bile leakage treated by ERCP and ENBD insertion. Four patients with hemobilia were detected by the peripheral blood and fecal occult 
blood test and become negative after 1-3 days treated by octreotide or hemostatic drugs. Just only 1 case in group A experienced acute biliary pancreatitis on the third day after surgery and cured with octreotide. The cholangitis and pneumonia were cured with antibiotics, and the wound infection was treated by dressing change. No patients in either group developed postoperative grade $\mathrm{C}$ bile leakage, retained or recurrent stones, jaundice, and bile duct stricture during the follow-up period.

\section{Discussion/Conclusion}

Since LCBDE was first performed by Philips et al. in 1993 [16], the managements of the bile duct diseases have changed radically in recent years. A large multicenter randomized controlled trial indicated that one-stage laparoscopic treatment was a better option for those patients with ASA I and II [17]. In addition, a comprehensive meta-analysis demonstrates that PDC after LCBDE is feasible for choledocholithiasis because it is associated with fewer complications than T-tube placement [3]. However, Estellés Vidagany et al. [4] reported that the overall complication rate of PDC was $15 \%$ and bile leakage was $6.8 \%$. Therefore, some scholars suggested that intraoperative ENBD should be retained after LCBDE + PDC to prevent the complications. Liao et al. [18] believed there was no significant difference in the operation time, the intraoperative blood loss, and the incidence of bile leakage between intraoperative ENBD and T-tube drainage. Furthermore, Yin et al. [19] thought that the postoperative complication rate of intraoperative ENBD was lower than that of preoperative ERCP but without significant difference ( 1.9 vs. $4.2 \%, p=0.29$ ). However, it is well known that serious complications of ENBD include acute pancreatitis, sepsis, bleeding, cholangitis, duodenal perforation, and so on $[5,6]$. Moreover, multivariable regression analysis indicated that slender CBD $(<0.8 \mathrm{~cm})$ and inexperienced surgeons were the high-risk factors for bile leakage after PDC [20]. So, it is still controversial whether intraoperative ENBD should be applied following LCBDE + PDC for all patients [7].

Initially, the main surgery principle of choledocholithiasis is to remove stones clearly to keep the CBD unobstructed. Any factor that is likely to cause obstruction of the distal bile duct will lead to high pressure in the duct and ischemia at the suture site, resulting in the increased chances of bile leakage and duct stricture [1]. In our study, preoperative imaging and intraoperative choledochoscopy provided direct visual information about stones or distal CBD obstruction to the surgeons. Patients who met the inclusion criteria were completed the laparoscopic surgical procedure with a rate of duct clearance of $100 \%$ (Table 1), which was consistent with other published series on LCBDE (88 100\%) [21-23].

The key factors to clear stones include the size and number of stones and the diameter of CBD. In our research, the cases according to the inclusion criteria were selected; that is, the size of stone must be $<1.5 \mathrm{~cm}$ [8], the number of stones must be $<5$ [19], and the diameter of CBD must be $>0.8 \mathrm{~cm}$ [1]. In group A, the mean stone size was $0.87 \pm 0.36 \mathrm{~cm}$, the mean number was $1.96 \pm 1.07$, and the mean diameter of CBD was $1.26 \pm 0.34 \mathrm{~cm}$ (Table 1 ). Multiple studies have shown that too many or too large stones may not be able to be reliably and safely removed through LCBDE. Meanwhile, the minimum diameter of $0.8 \mathrm{~cm}$ is essential to perform safe choledochotomy and stone removal as well as to avoid postoperative CBD stricture $[3,19,24]$.

In addition, the intraoperative findings and postoperative recovery were compared between the 2 groups. Group A had the advantages of shorter operation time, less bleeding, faster recovery, and lower hospital cost $(p<0.05)$ (Table 2$)$. The average operation time of group A was shorter because of the time saved by not having to place an ENBD. Furthermore, intraoperative ENBD through ERCP led to higher hospital cost and longer postoperative hospital stay. 
The overall rate of postoperative complication and bile leakage was 14.81 and $4.94 \%$ in group A, compared with 16.87 and $2.41 \%$ in group B ( $p=0.719 ; p=0.440)$, respectively (Table 3). The differences between the 2 groups were not statistically significant. In addition, the results of this study are consistent with existing literature, which shows 15 and $6.8 \%$, respectively [4]. Furthermore, we classified the reasons for bile leakage after PDC without ENBD as follows: (1) improper patient selection, (2) retained stones, (3) inexperience with laparoscopic suturing techniques, and (4) CBD injury. In our study, all bile leakage stopped spontaneously through interventional or conservative treatment without reoperation. Moreover, there was no severe bile leakage (grade C), recurrent stones, or bile duct stricture. These data proved that PDC without ENBD did not increase the risk of postoperative complications.

In conclusion, under the strictly screening conditions, PDC after LCBDE without ENBD has much shorter operative time, less bleeding, cheaper cost, and quicker recovery than that with ENBD, in addition to no increase in the morbidity rate of operative complications. So, PDC after LCBDE without ENBD in appropriately selected patients is safe and effective and worthy of clinical application.

\section{Acknowledgement}

We acknowledge Professor Ling-Hui Gao (Department of Hepatobiliary Pancreatic Surgery, the First Affiliated Hospital of Hainan Medical University, Haikou, Hainan, China) for insightful review of this manuscript.

\section{Statement of Ethics}

This research was conducted ethically in accordance with the World Medical Association Declaration of Helsinki. The ethical approval was obtained from the First Affiliated Hospital of Hainan Medical University Ethics Committee (Grant No. 2016 [scientific research] No [3]), and written informed consents were obtained from all patients.

\section{Conflict of Interest Statement}

All authors declare that there are no conflicts of interest to disclose.

\section{Funding Sources}

This work was funded by the National Natural Science Foundation of China (No. 81860514 to Professor Yan Yang) and the Natural Science Foundation of Hainan (No. 2019RC380 to Professor Yan Yang).

\section{Author Contributions}

The work presented here was carried out in collaboration with all authors. Yan Yang defined the research theme and designed the research method. Yan Yang, Lin Han, and Da-Ning Lin performed the laparoscopic surgical procedures. Zeng-Ji Hu performed ERCP surgical procedures to place the ENBD. Da-Ning Lin, Wei Tu, Feng Chen, and Yong-Qiang Li were responsible for perioperative management and clinical data collection. Yan Yang and Wei Tu oversaw the statistics and analysis of clinical data. Feng Chen and YongQiang Li wrote the paper. All authors have contributed to and approved the paper. 


\section{References}

1 Wen SQ, Hu QH, Wan M, Tai S, Xie XY, Wu Q, et al. Appropriate patient selection is essential for the success of primary closure after laparoscopic common bile duct exploration. Dig Dis Sci. 2017 May;62(5):1321-6.

2 Khaled YS, Malde DJ, de Souza C, Kalia A, Ammori BJ. Laparoscopic bile duct exploration via choledochotomy followed by primary duct closure is feasible and safe for the treatment of choledocholithiasis. Surg Endosc. 2013 Nov;27(11):4164-70.

3 Podda M, Polignano FM, Luhmann A, Wilson MS, Kulli C, Tait IS. Systematic review with meta-analysis of studies comparing primary duct closure and T-tube drainage after laparoscopic common bile duct exploration for choledocholithiasis. Surg Endosc. 2016 Mar;30(3):845-61.

4 Estellés Vidagany N, Domingo Del Pozo C, Peris Tomás N, Díez Ares JÁ, Vázquez Tarragón A, Blanes Masson F. Eleven years of primary closure of common bile duct after choledochotomy for choledocholithiasis. Surg Endosc. 2016 May;30(5):1975-82.

5 Park SY, Park CH, Cho SB, Yoon KW, Lee WS, Kim HS, et al. The safety and effectiveness of endoscopic biliary decompression by plastic stent placement in acute suppurative cholangitis compared with nasobiliary drainage. Gastrointest Endosc. 2008 Dec;68(6):1076-80.

6 Noji T, Nakamura F, Nakamura T, Kato K, Suzuki O, Ambo Y, et al. ENBD tube placement prior to laparoscopic cholecystectomy may reduce the rate of complications in cases with predictably complicating biliary anomalies. J Gastroenterol. 2011 Jan;46(1):73-7.

7 Hua J, Lin S, Qian D, He Z, Zhang T, Song Z. Primary closure and rate of bile leak following laparoscopic common bile duct exploration via choledochotomy. Dig Surg. 2015;32(1):1-8.

8 Yin Z, Xu K, Sun J, Zhang J, Xiao Z, Wang J, et al. Is the end of the T-tube drainage era in laparoscopic choledochotomy for common bile duct stones is coming? A systematic review and meta-analysis. Ann Surg. 2013 Jan; 257(1):54-66.

9 Yi HJ, Hong G, Min SK, Lee HK. Long-term outcome of primary closure after laparoscopic common bile duct exploration combined with choledochoscopy. Surg Laparosc Endosc Percutan Tech. 2015 Jun;25(3):250-3.

10 Leida Z, Ping B, Shuguang W, Yu H. A randomized comparison of primary closure and T-tube drainage of the common bile duct after laparoscopic choledochotomy. Surg Endosc. 2008;22(7):1595-600.

11 Abellán Morcillo I, Qurashi K, Abrisqueta Carrión J, Martinez Isla A. Laparoscopic common bile duct exploration. Lessons learned after 200 cases. Cir Esp. 2014 May;92(5):341-7.

12 Quaresima S, Balla A, Guerrieri M, Campagnacci R, Lezoche E, Paganini AM. A 23 year experience with laparoscopic common bile duct exploration. HPB. 2017 Jan;19(1):29-35.

13 Dindo D, Demartines N, Clavien PA. Classification of surgical complications: a new proposal with evaluation in a cohort of 6336 patients and results of a survey. Ann Surg. 2004 Aug;240(2):205-13.

14 Koch M, Garden OJ, Padbury R, Rahbari NN, Adam R, Capussotti L, et al. Bile leakage after hepatobiliary and pancreatic surgery: a definition and grading of severity by the International Study Group of Liver Surgery. Surgery. 2011 May;149(5):680-8.

15 Saharia PC, Zuidema GD, Cameron JL. Primary common duct stones. Ann Surg. 1977 May;185(5):598-604.

16 Phillips EH, Carroll BJ, Pearlstein AR, Daykhovsky L, Fallas MJ. Laparoscopic choledochoscopy and extraction of common bile duct stones. World J Surg. 1993 Jan-Feb;17(1):22-8.

17 Cuschieri A, Lezoche E, Morino M, Croce E, Lacy A, Toouli J, et al. E.A.E.S. multicenter prospective randomized trial comparing two-stage vs single-stage management of patients with gallstone disease and ductal calculi. Surg Endosc. 1999 Oct;13(10):952-7.

18 Liao NS, Lin F, Xin DY. Clinical application of endoscopic nasobiliary drainage in laparoscopic common bile duct exploration and primary suture. China J Endosc. 2016;22(1):28-31.

19 Yin P, Wang M, Qin R, Zhang J, Xiao G, Yu H, et al. Intraoperative endoscopic nasobiliary drainage over primary closure of the common bile duct for choledocholithiasis combined with cholecystolithiasis: a cohort study of 211 cases. Surg Endosc. 2017 Aug;31(8):3219-26.

20 Liu D, Cao F, Liu J, Xu D, Wang Y, Li F. Risk factors for bile leakage after primary closure following laparoscopic common bile duct exploration: a retrospective cohort study. BMC Surg. 2017 Jan 05;17(1):1-8.

21 Noble H, Tranter S, Chesworth T, Norton S, Thompson M. A randomized, clinical trial to compare endoscopic sphincterotomy and subsequent laparoscopic cholecystectomy with primary laparoscopic bile duct exploration during cholecystectomy in higher risk patients with choledocholithiasis. J Laparoendosc Adv Surg Tech A. 2009 Dec;19(6):713-20.

22 Rogers SJ, Cello JP, Horn JK, Siperstein AE, Schecter WP, Campbell AR, et al. Prospective randomized trial of LC+LCBDE vs ERCP/S+LC for common bile duct stone disease. Arch Surg. 2010 Jan;145(1):28-33.

23 Zhang HW, Chen YJ, Wu CH, Li WD. Laparoscopic common bile duct exploration with primary closure for management of choledocholithiasis: a retrospective analysis and comparison with conventional T-tube drainage. Am Surg. 2014 Feb;80(2):178-81.

24 Cai Y, Liu X. Laparoscopic choledochotomy followed by primary duct closure for choledocholithiasis. Surg Endosc. 2014 May;28(5):1750-1. 